\title{
Stellar populations of stellar halos: Results from the Illustris simulation
}

\author{
B. A. Cook, C. Conroy, A. Pillepich and L. Hernquist \\ Harvard-Smithsonian Center for Astrophysics \\ 60 Garden St., Cambridge, MA 02138 \\ Contact email: bcook@cfa.harvard.edu
}

\begin{abstract}
The influence of both major and minor mergers is expected to significantly affect gradients of stellar ages and metallicities in the outskirts of galaxies. Measurements of observed gradients are beginning to reach large radii in galaxies, but a theoretical framework for connecting the findings to a picture of galactic build-up is still in its infancy. We analyze stellar populations of a statistically representative sample of quiescent galaxies over a wide mass range from the Illustris simulation. We measure metallicity and age profiles in the stellar halos of quiescent Illustris galaxies ranging in stellar mass from $10^{10}$ to $10^{12} M_{\odot}$, accounting for observational projection and luminosity-weighting effects. We find wide variance in stellar population gradients between galaxies of similar mass, with typical gradients agreeing with observed galaxies. We show that, at fixed mass, the fraction of stars born in-situ within galaxies is correlated with the metallicity gradient in the halo, confirming that stellar halos contain unique information about the build-up and merger histories of galaxies.
\end{abstract}

\section{Introduction}

Stellar halos are diffuse regions of stars ubiquitously found surrounding galaxies (with a few notable exceptions, van Dokkum et al. 2014). They are observed to extend to many times a galaxy's effective radius (Martínez-Delgado et al. 2010), where dynamical timescales are very long compared to the ages of their host galaxies (Eggen et al. 1962). Detections of streams and tidal features in integrated light images of the halos in the Milky Way (Helmi et al. 1999), Andromeda (Ibata et al. 2001), and external galaxies (Martinez-Delgado et al. 2014) imply that stellar halos result from mergers and accretion as galaxies grow hierarchically, a picture anticipated by numerical simulations (e.g., Johnston et al. 1996; Bullock \& Johnston 2005; Cooper et al. 2010; Pillepich et al. 2014). The long timescales at large radius can help preserve the information content of each galaxy's merger history, which has been used as motivation for observations of kinematics and populations of the stellar halo (Bell et al. 2008; Schlaufman et al. 2009).

Stellar populations - characterized by the metallicities and ages of stars - are clues to when and in what systems stars originally formed (Greene et al. 2015). The massmetallicity relation in galaxies (Tremonti et al. 2004), combined with hierarchical accretion, implies that metallicity gradients should indicate the relative contributions of more-or-less massive progenitor systems to particular regions of a galaxy and its halo. Different merger histories should leave distinct imprints on observed stellar population gradients (Hirschmann et al. 2015), although stars formed in-situ, or within the galaxy in which they now reside, may still be significant in the inner regions of the halo (Font et al. 2011). For massive, early-type galaxies (usually red, quiescent, and elliptical), the twophase formation scenario outlines the contributions of mergers and in-situ star formation to the formation of a metallicity gradient (Spolaor et al. 2010; Pastorello et al. 2014). The early phase of dissipative collapse leads to an steep, negative gradient from in-situ 
stars, while the later phase involving accretion of stars from smaller satellite galaxies tends to flatten these gradients (Kobayashi 2004).

Observations of stellar population gradients are beginning to accumulate for large samples of galaxies (Spolaor et al. 2010; Pastorello et al. 2014) and for individual giant ellitpicals (Coccato et al. 2010, 2011), and they are reaching continually larger radii through integral-field spectroscopy (Delgado et al. 2015; Greene et al. 2015). Observations find a wide variety of gradients between galaxies with similar masses and morphologies, which is to be expected if particular merger histories shape the gradients in a stochastic way. Unfortunately, the observational need for detailed predictions connecting gradients to merger histories have so far outpaced numerical simulations. Due to limitations in computing resources, hydrodynamical simulations have so far only resolved stellar halo populations around individual galaxies (Abadi et al. 2006; Hirschmann et al. 2015; Cooper et al. 2015), a method which lacks the statistical power required to replicate the observed halo-to-halo variations. Large samples of galaxy halos have been produced in N-body simulations, some of which include semi-analytic models and stellar-tagging techniques (Cooper et al. 2010), but these do not include baryonic physics effects, which may shape the dark matter distribution, and must rely on complicated fitting functions to generate "stellar particles" with realistic orbital properties (Bailin et al. 2014).

In this work, we measure stellar population gradients in a sample of quiescent galaxies from the hydrodynamical cosmological simulation Illustris. With the statistical power of Illustris' large cosmological volume and its self-consistent model for star and galaxy formation, we are able to match the observed galaxy-to-galaxy variance in gradients and show that these variations are indeed reflective of different galactic merger histories.

\section{Methods}

\subsection{The Simulations}

The Illustris simulations (Vogelsberger et al. 2014a; Genel et al. 2014; Nelson et al. 2015) are a suite of N-body+hydrodynamical cosmological simulations (106.5 Mpc on a side), run at multiple resolutions with the adaptive mesh code AREPO (Springel 2010; Vogelsberger et al. 2013). The simulations model key physical processes for the formation of galaxies, including stellar formation, evolution, and feedback, chemical enrichment, radiative cooling, supermassive black hole growth, and feedback from AGN. The highest resolution run (Illustris-1, hereafter simply Illustris) has a mass resolution of $m_{D M}=$ $6.26 \times 10^{6} M_{\odot}$ and $m_{\text {baryon }} \sim 1.26 \times 10^{6} M_{\odot}$ for the dark matter and baryonic components, respectively. At $z=0$ gravitational forces for stellar particles are resolved to a softening length of $0.7 \mathrm{kpc}$. Illustris was run from $z=127$ to $z=0$ using $\Lambda$ CDM cosmological paramters consistent with WMAP9 $\left(\Omega_{\Lambda}=0.7274, \Omega_{m}=0.2726, h=0.704\right.$, Hinshaw et al. 2013).

At $z=0$, the Illustris volume contains more than $4 \times 10^{4}$ well-resolved galaxies (Vogelsberger et al. 2014a), with a reasonable diversity of morphologies and colors, including early-type and late-type galaxies (Torrey et al. 2015). The most massive central galaxies (as identified by the FOF and SUBFIND algorithms, Springel et al. 2001; Dolag et al. 2009) reproduced in the simulation have stellar masses within their stellar half-mass radii of $M_{*} \sim 1-2 \times 10^{12} M_{\odot}$. The simulation reproduces the observed $z=0$ mass-metallicity relation in galaxies (Vogelsberger et al. 2014b), and a reasonable relation between galaxy mass and stellar ages once luminosity weighting is properly taken into account. 


\subsection{The Quiescent Galaxy Sample}

Our goal to reproduce measurements in the outer regions of early-type galaxies motivates the selection criteria of our sample. We select central galaxies (which are not satellites/subhalos of more massive parents) with stellar masses $M_{*} \geqslant 10^{10} M_{\odot}^{\dagger}$. Each galaxy in this mass range is resolved with at least a few hundred star particles beyond 8 effective radii, ensuring that we constrain gradients all the way throughout the low-density outer regions and up to 10 times the effective radius.

In this work, we examine the properties of simulated quiescent galaxies, in comparison to observed early-type galaxies. Of all Illustris galaxies with $M_{*}>10^{10} M_{\odot}$, we select the 352 quiescent galaxies with specific star formation rates SSFR $\leqslant 10^{-11.5} \mathrm{yr}^{-1}$ within twice the stellar half-mass radius. See Cook et al. (In Prep.) for details.

\subsection{Fitting Stellar Population Gradients}

We simulate observational projection effects by projecting the radii of star particles from the center of their host galaxies against a random line-of-sight. We also account for observational biases by weighting the contribution of each star particle relative to its V-band lumionsity ${ }^{\ddagger}$. Star particles identified by SUBFIND as bound to smaller subhalos or satellites are not included in the analysis.

Using these observational considerations, we measure the 2-D azimuthally-averaged values of stellar metallicity and age in five logarithmically-spaced radial bins over a chosen projected radius range. We focus on three particular ranges in units of the $\mathrm{V}$-band effective radius $\left(R_{e} \mathbf{\Phi}\right)$, which we label: the inner galaxy $\left(0.1-1 R_{e}\right)$, the outer galaxy (1 $\left.2 R_{e}\right)$, and the stellar halo $\left(2-10 R_{e}\right)$. We then calculate a logarithmic gradient by fitting a line to these averages, equally weighting each radius bin: $f(r)=f\left(R_{e}\right)+\nabla_{f} \log _{10}\left(r / R_{e}\right)$, with $f$ the mean log-metallicity $([\mathrm{Z} / \mathrm{H}])$ or the mean age (in Gyrs) in each bin.

\section{Results and Implications}

\subsection{Comparisons to Observed Gradients}

The measured metallicity and age gradients in the inner and outer galaxy ranges $(<$ $\left.2 R_{e}\right)$ are shown as a function of central velocity dispersion $\left(\sigma_{0}\right.$, within $\left.\frac{1}{8} R_{e}\right)$ in Fig. 1 . Observations can constrain gradients in these radius ranges for large samples of galaxies (Spolaor et al. 2010; Pastorello et al. 2014; Greene et al. 2015; Delgado et al. 2015), but so far there are only a few individual cases for comparison in the stellar halo $\left(>2 R_{e}\right)$ range. We compare our measurements to observations of individual galaxies from Spolaor et al. (2010) (inner galaxy) and stacked measurements by Greene et al. (2015) (outer galaxy).

The typical values of our metallicity and age gradients agree with observations: metallicity gradients are negative - outer regions are more metal poor - while age gradients are roughly flat - inner and outer regions have similar average ages. Of particular importance is the fact that our measurements also reproduce the observed scatter in gradients between galaxies of similar masses. Illustris, with its large statistical sampling of galaxies, is uniquely able to replicate this galaxy-galaxy variance, whereas more individualized simulations cannot replicate the wide variety of observed gradients.

$\dagger$ Throughout the paper, $M_{*}$ denotes the total mass in stars within the stellar half-mass radius $\left(R_{\text {half }}\right)$ after removing the contributions of gravitationally-bound satellites identified by SUBFIND.

$\ddagger$ Star particles in each galaxy are assigned luminosities in several common observational bands using single-age stellar population SED templates (Torrey et al. 2015).

I Each galaxy's effective radius is the projected radius within which one-half of its total $\mathrm{V}$-band luminosity is located. This is calculated as an average over 100 random lines of sight. 

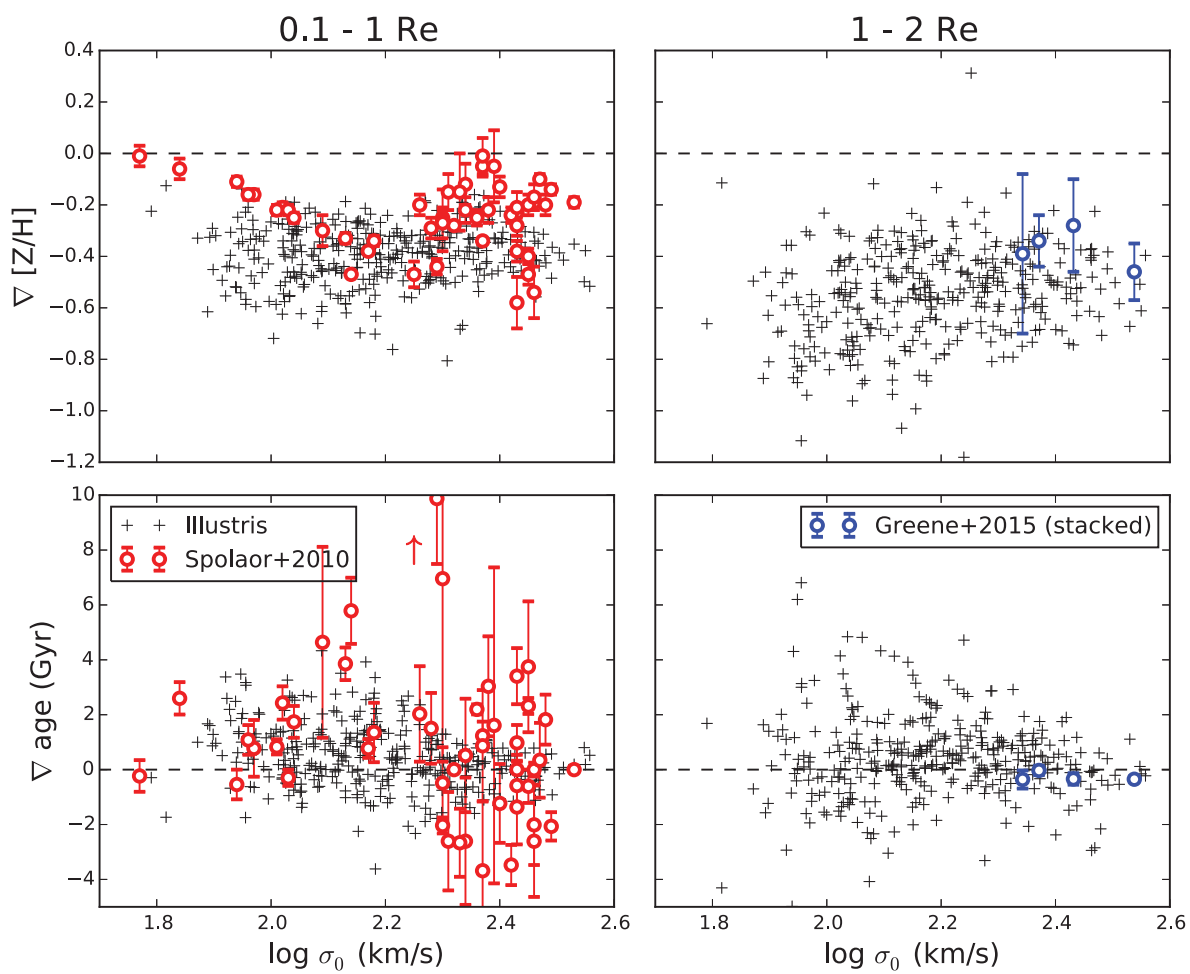

Figure 1. Measurements of metallicity (top) and age (bottom) gradients in Illustris quiescent galaxies (black cross marks). These are calculated in the inner $\left(0.1-1 R_{e}\right.$, left $)$ and outer $(1-$ $2 R e$, right) galaxy ranges, and include projection along a random line of sight and luminosity-weighting. We compare to the measurements of Spolaor et al. (2010) and Greene et al. (2015) in the inner and outer galaxy, respectively.

Measured stellar populations in the halo of NGC 4889 (Coccato et al. 2010), the Brightest Central Galaxy (BCG) of the Coma cluster, show a similarly steep metallicity gradient to $1.2 \mathrm{R}_{e}$, but a shallower gradient from $1.2-4 \mathrm{R}_{e}(-0.1 \pm 0.2)$ than typical for the most massive Illustris galaxies. A flat metallicity gradient is also found in the halo of NGC 3311, the BCG of the Hydra I cluster (Coccato et al. 2011), although there are signatures of significant substructure. Illustris has only a few galaxies in the regime of these most massive systems, and future simulations with larger volumes should provide improved statistics at this massive end.

\subsection{Relating Metallicity Gradients to Merger Properties}

Here, we study whether the scatter in stellar population gradients among Illustris galaxies at fixed mass - which matches the scatter observed in early-type galaxies - can be connected to the particular merger histories of each galaxy. We quantify the contribution of mergers to a galaxy's build-up in terms of the in-situ fraction: the mass fraction of a galaxy's stars which were formed within the galaxy or its main progenitor branch. Galaxies with low in-situ fractions were primarily built-up from mergers and accretion of smaller systems, while galaxies with high in-situ fractions have had little influence from mergers.

In the hierarchical model of galaxy formation, galaxies grow continually through mergers with increasingly massive neighbors. This effect, reproduced in many cosmological simulations and seen clearly in Illustris (Rodriguez-Gomez et al. 2015), results in a strong 

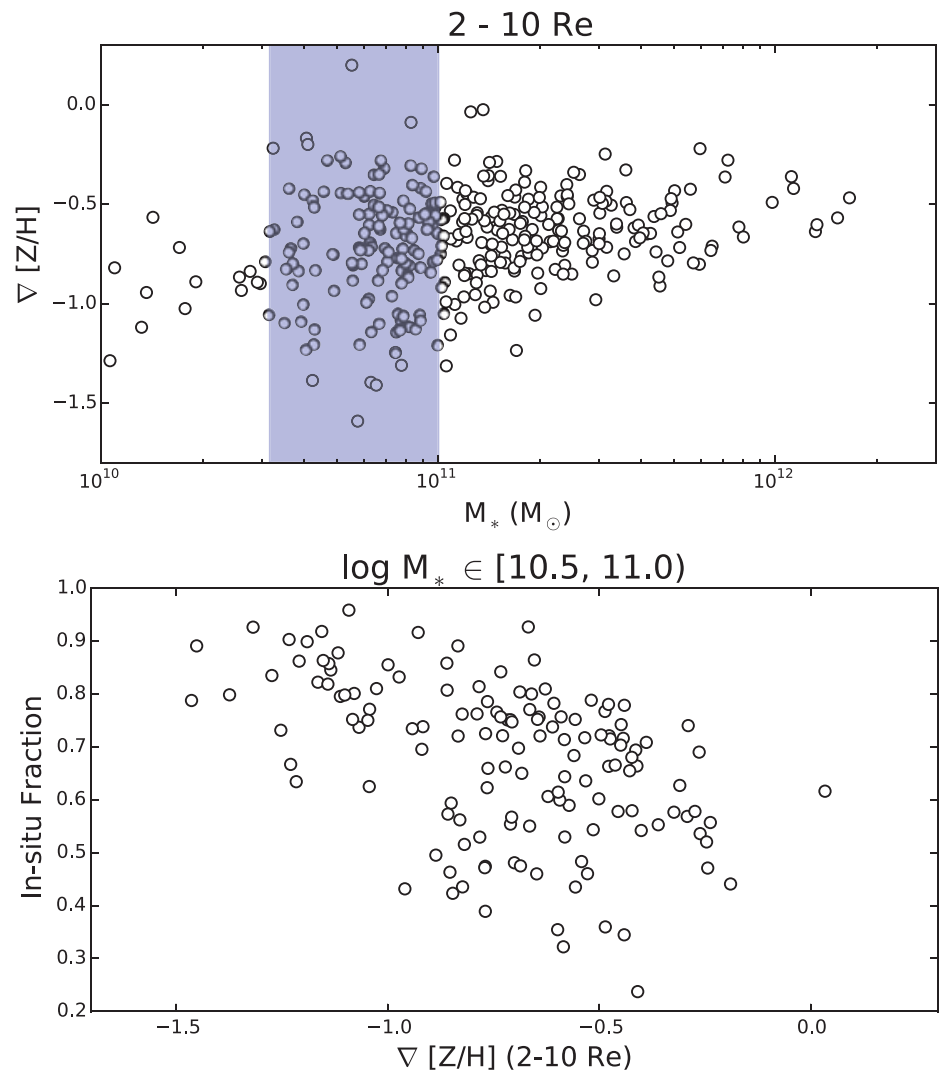

Figure 2. Top: The metallicity gradient in the stellar halo $\left(2-10 R_{e}\right)$ of the quiescent Illustris sample, versus galaxy stellar mass. As at smaller radii, the metallicity gradient shows wide galaxy-galaxy variance within narrow mass ranges, such as between $10^{10.5}$ and $10^{11} M_{\odot}$ (shaded region). Bottom: The correlation between galaxy in-situ fraction and stellar halo metallicity gradient, for galaxies between in the mass range given above. Galaxies with lower in-situ fractions (mergers more significant in growth) have flatter metallicity gradients.

trend towards lower in-situ fractions in more massive galaxies. To disentangle this effect from the individual histories, we can focus our analysis to small ranges in stellar mass. Over a sufficiently narrow window, mass differences are small enough that the in-situ fraction should be driven primarily by the particular merger history of each galaxy.

In Fig. 2, we show the metallicity gradient in the stellar halo $\left(2-10 R_{e}\right)$. Galaxies have overall negative metallicity gradients and there is a large scatter at fixed mass, just as in the inner and outer galaxy regions. When selecting galaxies in the narrow mass window from $10^{10.5}$ to $10^{11} M_{\odot}$ and comparing to the in-situ fraction, we see that there is a correlation. Low in-situ fractions are associated with flatter metallicity gradients in the stellar halo, while galaxies with higher in-situ fractions have steeper halo gradients.

This result suggests that much of the scatter in observed metallicity gradients can be explained through a variety of galactic merger histories. The process of building-up a galaxy through mergers appears to flatten the metallicity gradient. Measuring gradients into the outer stellar halo should provide clues about the relative importance of mergers in a particular early-type galaxy's evolution (see also, Pillepich et al. 2014).

We note that this correlation is significant only in the stellar halo regions $\left(2-10 R_{e}\right)$ of our simulated galaxies. Metallicity gradients in the inner and outer galaxy regions 
$\left(<2 R_{e}\right)$ show no association with the in-situ fraction. This emphasizes the importance of measuring gradients into the outer stellar halo (well beyond $2 R_{e}$ ), where the significantly longer dynamical timescales result in more lasting imprints from mergers. Stellar population gradients in the halo retain the information content of merger histories; it is still unclear what leads to the wide scatter in gradients in the interior regions.

\section{Acknowledgements}

This material is based upon work supported by the NSF Graduate Research Fellowship Program under Grant No. DGE1144152.

\section{References}

Abadi, M. G., Navarro, J. F., \& Steinmetz, M. 2006, http://dx.doi.org/10.1111/ j.1365-2966.2005.09789.x Mon. Not. R. Astron. Soc., 365, 747

Bailin, J., Bell, E. F., Valluri, M., et al. 2014, http://dx.doi.org/10.1088/0004-637X/783/2/ 95 Astrophys. J., 783, 95

Bell, E. F., Zucker, D. B., Belokurov, V., et al. 2008, http://dx.doi.org/ 10.1086/588032 Astrophys. J., 680, 295

Bullock, J. S. \& Johnston, K. V. 2005, http://dx.doi.org/10.1086/497422 Astrophys. J., 635, 931

Coccato, L., Gerhard, O., \& Arnaboldi, M. 2010, http://dx.doi.org/10.1111/j.1745-3933. 2010.00897.x Mon. Not. R. Astron. Soc. Lett., 407, L26

Coccato, L., Gerhard, O., Arnaboldi, M., \& Ventimiglia, G. 2011, http://dx.doi.org/ 10.1051/0004-6361/201117546 Astron. Astrophys., 533, A138

Cooper, A. P., Parry, O. H., Lowing, B., Cole, S., \& Frenk, C. 2015, http://arxiv.org/ abs/1501.04630eprint, http://arxiv.org/abs/1501.04630arXiv:1501.04630

Cooper, A. P., Cole, S., Frenk, C. S., et al. 2010, http://dx.doi.org/10.1111/ j.1365-2966.2010.16740.x Mon. Not. R. Astron. Soc., 406, 744

Delgado, R. M. G., García-Benito, R., Pérez, E., et al. 2015, http://arxiv.org/ abs/1506.04157eprint arXiv:1506.04157, 42

Dolag, K., Borgani, S., Murante, G., \& Springel, V. 2009, http://dx.doi.org/ 10.1111/j.1365-2966.2009.15034.x Mon. Not. R. Astron. Soc., 399, 497

Eggen, O. J., Lynden-Bell, D., \& Sandage, A. R. 1962, http://dx.doi.org/10.1086/147433 Astrophys. J., 136, 748

Font, A. S., McCarthy, I. G., Crain, R. A., et al. 2011, http://dx.doi.org/10.1111/ j.1365-2966.2011.19227.x Mon. Not. R. Astron. Soc., 416, 2802

Genel, S., Vogelsberger, M., Springel, V., et al. 2014, http://dx.doi.org/ 10.1093/mnras/stu1654 Mon. Not. R. Astron. Soc., 445, 175

Greene, J. E., Janish, R., Ma, C.-P., et al. 2015, http://dx.doi.org/10.1088/0004-637X/807/ 1/11 Astrophys. J., 807, 11

Helmi, A., White, S. D. M., de Zeeuw, P. T., \& Zhao, H. 1999, http://dx.doi.org/ 10.1038/46980 Nature, Vol. 402, Issue 6757, pp. 53-55 (1999).

Hinshaw, G., Larson, D., Komatsu, E., et al. 2013, http://dx.doi.org/10.1088/0067-0049/ 208/2/19 Astrophys. J. Suppl. Ser., 208, 19

Hirschmann, M., Naab, T., Ostriker, J. P., et al. 2015, http://dx.doi.org/ 10.1093/mnras/stv274 Mon. Not. R. Astron. Soc., 449, 528

Ibata, R., Irwin, M., Lewis, G., Ferguson, A. M. N., \& Tanvir, N. 2001, http://labs.adsabs.harvard.edu/adsabs/abs/2001Natur.412..49I/ Nature, Vol. 412, Issue 6842, pp. 49-52 (2001).

Johnston, K. V., Hernquist, L., \& Bolte, M. 1996, http://dx.doi.org/10.1086/177418 Astrophys. J., 465, 278

Kobayashi, C. 2004, http://dx.doi.org/10.1111/j.1365-2966.2004.07258.x Mon. Not. R. Astron. Soc., 347, 740

Martinez-Delgado, D., D'Onghia, E., Chonis, T. S., et al. 2014, http://adsabs.harvard. edu/abs/2014arXiv1410.6368Meprint, http://arxiv.org/abs/1410.6368arXiv:1410.6368 
Martínez-Delgado, D., Gabany, R. J., Crawford, K., et al. 2010, http://dx.doi.org/ 10.1088/0004-6256/140/4/962 Astron. J., 140, 962

Nelson, D., Pillepich, A., Genel, S., et al. 2015, http://arxiv.org/abs/1504.00362eprint arXiv:1504.00362, http://arxiv.org/abs/1504.00362arXiv:1504.00362

Pastorello, N., Forbes, D. A., Foster, C., et al. 2014, http://dx.doi.org/10.1093/mnras/stu937 Mon. Not. R. Astron. Soc., 442, 1003

Pillepich, A., Vogelsberger, M., Deason, A., et al. 2014, http://dx.doi.org/10.1093/ mnras/stu1408 Mon. Not. R. Astron. Soc., 444, 237

Rodriguez-Gomez, V., Genel, S., Vogelsberger, M., et al. 2015, http://dx.doi.org/10.1093/ mnras/stv264 Mon. Not. R. Astron. Soc., 449, 49

Schlaufman, K. C., Rockosi, C. M., Prieto, C. A., et al. 2009, http://dx.doi.org/10.1088/ 0004-637X/703/2/2177 Astrophys. J., 703, 2177

Spolaor, M., Kobayashi, C., Forbes, D. A., Couch, W. J., \& Hau, G. K. T. 2010, http://dx.doi.org/10.1111/j.1365-2966.2010.17080.x Mon. Not. R. Astron. Soc., 408, 272

Springel, V. 2010, http://dx.doi.org/10.1111/j.1365-2966.2009.15715.x Mon. Not. R. Astron. Soc., 401, 791

Springel, V., White, S. D. M., Tormen, G., \& Kauffmann, G. 2001, http://dx.doi.org/ 10.1046/j.1365-8711.2001.04912.x Mon. Not. R. Astron. Soc., 328, 726

Torrey, P., Snyder, G. F., Vogelsberger, M., et al. 2015, http://dx.doi.org/ 10.1093/mnras/stu2592 Mon. Not. R. Astron. Soc., 447, 2753

Tremonti, C. A., Heckman, T. M., Kauffmann, G., et al. 2004, http://dx.doi.org/10.1086/ 423264 Astrophys. J., 613, 898

van Dokkum, P. G., Abraham, R., \& Merritt, A. 2014, http://dx.doi.org/10.1088/ 2041-8205/782/2/L24 Astrophys. J., 782, L24

Vogelsberger, M., Genel, S., Sijacki, D., et al. 2013, http://dx.doi.org/10.1093/mnras/ stt1789 Mon. Not. R. Astron. Soc., 436, 3031

Vogelsberger, M., Genel, S., Springel, V., et al. 2014a, http://dx.doi.org/10.1093/mnras/ stu1536 Mon. Not. R. Astron. Soc., 444, 1518

-. 2014b, http://dx.doi.org/10.1038/nature13316 Nature, 509, 177 